\title{
Our Approach for Optimizing Vocal Outcomes in Transoral Laser Microsurgical Resection of Early Glottic Carcinoma
}

\author{
${ }^{1}$ Nupur K Nerurkar, ${ }^{2}$ Shraddha Deshmukh
}

\begin{abstract}
Objectives: To assess oncologic and functional outcomes of early glottic cancer cases treated with transoral laser microsurgical resection (TLMR) and to outline the principles for optimizing vocal outcomes in these cases.
\end{abstract}

Materials and methods: Sixty six patients with early glottic cancer (7 CIS, 43 T1a, 15 T1b, 1 T2) underwent TLMR. Postoperatively, voice quality was assessed by Oates Russell Voice Profile.

Results: Local control was achieved in $95.08 \%$ of the cases and larynx was preserved in $98.3 \%$. Residual disease developed in 3 patients. There were eight local recurrences. Single patient of $\mathrm{T} 1 \mathrm{~b}$ lesion was lost to follow-up and came back with stridor and underwent total laryngectomy.

Conclusion: Early glottic cancers can be effectively treated by TLMR with good oncologic outcome. Depth of infiltration of lesion is the most critical factor affecting the postoperative vocal outcome. In our study near-normal conversational voice to moderate change in voice was found after type I, II, and III cordectomies. Surprisingly, type VI cordectomy also gave a serviceable vocal outcome with moderate change in voice. In our opinion, type IV cordectomy does not give a serviceable vocal outcome even if performed unilaterally. Thus radiotherapy should be strongly considered in such cases for better vocal outcome.

Keywords: Carcinoma, Glottic, Laser, Voice.

How to cite this article: Nerurkar NK, Deshmukh S. Our Approach for Optimizing Vocal Outcomes in Transoral Laser Microsurgical Resection of Early Glottic Carcinoma. Int J Phonosurg Laryngol 2016;6(2):68-72.

Source of support: Nil

Conflict of interest: None

\section{INTRODUCTION}

The principles of laryngeal cancer management have greatly evolved in the past decade. The most common site of involvement is the glottis, which accounts for $75 \%$

\footnotetext{
${ }^{1}$ Consultant Laryngologist, ${ }^{2}$ Former Clinical Fellow

1,2Department of ENT, Bombay Hospital, Mumbai, Maharashtra India

Corresponding Author: Nupur K Nerurkar, Consultant Laryngologist, Department of ENT, Bombay Hospital, Mumbai Maharashtra, India, Phone: +919821034085, e-mail: nupurkapoor @yahoo.com
}

of the laryngeal cancer cases. ${ }^{1}$ Most of the patients of glottic cancer present early, i.e., without vocal fold fixation, nodal involvement, or extension beyond the larynx. ${ }^{2}$ In today's modern communication age, voice is critical and a radical surgery like total laryngectomy for an early glottic carcinoma is clearly an overkill. There has been a major shift of approach from radical surgery to the more conservative approaches which do not compromise on the oncological outcome and still preserve the laryngeal function. Furthermore, the use of laser has improved the surgeon's dexterity and the ability to work in confined spaces and has allowed precise removal of tissues with minimum damage to surrounding structures.

We describe here our protocol to maximize the vocal outcome measures in transoral laryngeal microsurgical resection of early glottic carcinoma while maintaining acceptable recurrence rates.

\section{MATERIALS AND METHODS}

In our study, patients of early glottic cancer (Tis, T1a, T1b, T2) treated with transoral laryngeal microsurgical resection (with or without laser) were analyzed retrospectively. A total of 90 patients with early glottic cancer were treated from January 2007 to November 2013 at Bombay Hospital, India. Data from 66 cases with a minimum follow-up of 12 months was analyzed, of which 65 patients were operated primarily and 1 patient was operated on after failure of radiotherapy. All patients were assessed with rigid/ flexible laryngoscopy. A computed tomography scan was advised for all the patients with suspected malignancy to assess the involvement of thyroid cartilage, anterior commissure, subglottic, or paraglottic space invasion. A TNM staging was done and patients included were Tis, T1, and T2 glottic lesions with no nodal metastasis (N0) and no distant metastasis (M0). Out of these, 7 (5 unilateral and 2 bilateral) (11\%) were carcinoma in situ, $43(65 \%)$ were $\mathrm{T} 1 \mathrm{a}, 15(23 \%)$ were $\mathrm{T} 1 \mathrm{~b}$, and $1(1 \%)$ was a T2 lesion. On histopathology, 63 patients had squamous cell carcinoma, 1 patient had adenocarcinoma, and 2 patients had sarcomatoid carcinoma.

\section{Procedure}

The depth of tumor infiltration was first determined by the subepithelial infiltration technique with 1 to $2 \mathrm{cc}$ 
of 1:10,000 saline adrenaline, which was injected in the Reinke's space. In cases where preoperative histopathology was not available, a biopsy was sent for frozen section examination. Lesions with depth of infiltration up to the superficial lamina propria were excised by the microflap technique keeping a 1 to $2 \mathrm{~mm}$ margin. Lesions with infiltration into the vocal ligament or the vocalis muscle were excised using the $\mathrm{CO}_{2}$ laser with 1 to $2 \mathrm{~mm}$ margin. The audit of depth of resection in our cases is described in Table 1. All the patients were counseled preoperatively that if intraoperatively the depth of invasion is up to more than half of the vocalis muscle, a total cordectomy is needed. This results in a poor postoperative voice with few options for medialization thyroplasty. Thus this surgery if needed will be abandoned and patient will be referred for radiotherapy for better voice outcomes.

\section{Follow-up}

Patients were followed up on 7th day, 1st month, and then every 2 months in the first 6 months. In the next 6 months they were reviewed 3 monthly. In the 2 nd year they were reviewed 6 monthly. Thereafter they were reviewed annually up to 5 years.

\section{RESULTS}

\section{Outcomes}

In our series, out of the total of 66 patients, 65 patients were treated primarily, 1 patient was operated as a postradiation failure. A single-stage surgery was done on 48 patients. A planned staged surgery was done in
1 patient. A total of 5 patients warranted a total cordectomy, which was not done to avoid poor vocal outcomes and thus these patients were referred for radiotherapy. Residual disease within 1 year of surgery was seen in 3 patients, out of which two opted for radiotherapy and one underwent a repeat laser resection. Recurrent disease after 1 year of surgery was seen in 8 patients and they underwent a repeat laser resection. Single patient of $\mathrm{T} 1 \mathrm{~b}$ lesion was lost to follow-up and came back with stridor and was salvaged by total laryngectomy.

Thus out of the 66 patients, 58 were treated only with endoscopic laser resection, 2 patients opted for radiotherapy for residual disease, 5 patients warranted a total cordectomy which was not done and these patients were referred for radiotherapy, and 1 patient who was lost to follow-up eventually required a total laryngectomy.

Thus laryngeal preservation was achieved in $98.3 \%$ of the cases and local control in $95.08 \%$.

\section{Hospitalization}

Most of the patients were hospitalized overnight and discharged the next day. The maximum duration of stay was 48 hours.

\section{Tracheostomy}

Tracheostomy was not required in any of the patients.

\section{Swallowing}

All the patients resumed solid feeds on the same day of the surgery. None of the patients complained of any aspiration or dysphagia.

Table 1: Type of cordectomy done (European Laryngology Society classification) with postcordectomy voice profile

\begin{tabular}{|c|c|c|c|c|c|}
\hline TNM staging & $\begin{array}{l}\text { Number } \\
\text { of cases }\end{array}$ & \multicolumn{2}{|c|}{ Site $U / L B / L$} & Type of cordectomy & $\begin{array}{l}\text { Postcordectomy voice (Oates Russell Voice } \\
\text { Profile) }\end{array}$ \\
\hline $\begin{array}{l}\text { Carcinoma } \\
\text { in situ }\end{array}$ & 7 & 5 & 2 & Type I in all & $\begin{array}{l}5(\mathrm{u} / \mathrm{L}) \text { : normal voice } 2(\mathrm{~b} / \mathrm{L}) \text { : mild change in } \\
\text { voice }\end{array}$ \\
\hline \multirow[t]{3}{*}{ T1a } & 43 & $43-$ & - & Type II: 35 & $\begin{array}{l}\text { Normal: } 19 \text {, mild change: } 11 \text {, mild to moderate } \\
\text { change: } 5\end{array}$ \\
\hline & & & & Type III: 5 & 2: Mild to moderate change \\
\hline & & & & $\begin{array}{l}3 \text { Cases would have required a Type } \\
\text { IV: cordectomy which was not done and } \\
\text { patient referred for radiotherapy }\end{array}$ & 3: Moderate change \\
\hline \multirow[t]{5}{*}{$\mathrm{T} 1 \mathrm{~b}$} & 15 & - & 15 & $\begin{array}{l}\text { Type I on one side and Type II on other } \\
\text { side- } 6\end{array}$ & 6: Mild to moderate \\
\hline & & & & Type II on both sides- 3 & 3: Mild to moderate \\
\hline & & & & $\begin{array}{l}\text { Type I on one side and Type III on other } \\
\text { side: } 1\end{array}$ & $\begin{array}{l}\text { 1: moderate to severe change (patient } \\
\text { underwent medialization thyroplasty following } \\
\text { which voice improved to mild change) }\end{array}$ \\
\hline & & & & Anterior commissurectomy, i.e., Type VI-3 & 3: Moderate change in voice \\
\hline & & & & $\begin{array}{l}2 \text { cases would have required a Type IV } \\
\text { cordectomy which was not done and } \\
\text { patient referred for radiotherapy }\end{array}$ & \\
\hline T2 & 1 & 1 & & Type II & Mild to moderate \\
\hline
\end{tabular}




\section{Quality of Voice}

Voice quality was assessed in all the patients at 12 weeks and was rated using the Oates Russell Voice Profile, which is a clinician-rated tool and was rated on a severity scale from normal to severe (Table 1).

\section{DISCUSSION}

The management of laryngeal cancers has undergone a multitude of changes in the past decade. Early glottic disease includes lesions ranging from carcinoma in situ to T2 lesions with normal vocal fold mobility. Treatment options for early glottic cancer include transoral microsurgery (with or without laser), radiotherapy, and open surgery. The main aim of treatment is to achieve total tumor control and secondly, to preserve the larynx with the best possible function. There is a longstanding dichotomy of opinion as to whether radiotherapy or surgery (with or without laser) is a superior treatment option.

A review of literature suggests that there is no statistically significant difference in the oncologic outcomes of external radiation and transoral laser surgical excision in the treatment of early-stage glottic cancer (Table 2). Similarly studies comparing survival outcomes in patients treated with radiotherapy $v s$ those treated with surgery, with or without laser, for early glottic cancer did not report any significant difference in overall or disease-free survival between the different treatment modalities. ${ }^{3-6}$

Thus it becomes clear that both the treatment modalities, i.e., radiotherapy as well as endoscopic microsurgical resection (with or without laser) confer similar cure rates and overall survival. Thus the choice of treatment should be decided keeping in mind post treatment voice outcome. This is dependent on the depth of tumor penetration and other tumor factors like the stage, site of lesion, and patient factors like age, general health, occupation, distance from the treatment center, and the expertise available.

The concept of transoral laryngeal microsurgery is not new and can be dated back to Lynch, who in 1915 described nine cases of endoscopic resection of early laryngeal cancers. Stutsman and McGavran ${ }^{8}$ in 1971 observed that 13 out of 60 hemilaryngectomy specimens of T1 glottic cancer showed no evidence of residual tumor following initial biopsy. Similar results were reported by Nassif et $\mathrm{al}^{9}$ who found $33 \%$ (5 out of 15 ) resection specimens to be free of tumor after previous biopsy. These studies indicate that hemilaryngectomy clearly was an overtreatment for early laryngeal cancers.

Strong and Jako ${ }^{10}$ in 1972 first described the use of $\mathrm{CO}_{2}$ laser for cancer larynx. These initial reports generated a lot of interest and Steiner ${ }^{11}$ in 1993 reported a series of 240 patients who underwent transoral laser microsurgery for carcinoma of the larynx. Among the 240 patients, 159 were with early glottic carcinomas (Tis 29, T1 96, T2a 34). Only 6\% developed a local recurrence with only one patient requiring a total laryngectomy. This pioneering work paved way for transoral laser microsurgery being accepted as a useful treatment method for early laryngeal cancer.

Compared to traditional open surgery techniques, transoral laryngeal microsurgery has distinct advantages namely shortened hospitalization stay, minimal morbidity, avoidance of tracheostomy, and early recovery of swallowing. In case if treatment with laser is inadequate, other salvage modalities in the form of radiotherapy and open surgery are still available. On the contrary, radiotherapy leads to longer duration of treatment, risks of radiation, and potential of causing irradiation induced malignancy in the young patient.

Table 2: Review of literature of studies comparing early glottic cancer patients treated with radiotherapy and surgery

\begin{tabular}{|c|c|c|c|c|c|}
\hline Study & $n$ & Stage & Treatment & Local control \% & Laryngeal preservation \% \\
\hline \multirow[t]{3}{*}{ Osborn et al ${ }^{3}$} & 57 & Tis, T1a & $\mathrm{RT}=34$ & RT: 91.2 & $\mathrm{RT}=94.1$ \\
\hline & & & TLM = 23 & TLM: 91.3 & $S X=100$ \\
\hline & & & & $p=0.72$ & $p=0.34$ \\
\hline \multirow[t]{4}{*}{ Mahler et $\mathrm{al}^{4}$} & 351 & T1a & $\mathrm{RT}=163$ & $\mathrm{RT}=95$ & Laryngectomies: \\
\hline & & & Laser SX $=188$ & $S X=92$ & $\mathrm{RT}: 9$ \\
\hline & & & & $p=0.395$ & Laser: 1 \\
\hline & & & & & $p=0.001^{*}$ \\
\hline \multirow[t]{3}{*}{ Schrijvers et al ${ }^{5}$} & 100 & T1a & $\mathrm{RT}=51$ & $\mathrm{RT}=73$ & $\mathrm{RT}=77$ \\
\hline & & & $\mathrm{CO}_{2}$ laser $=49$ & $S X=71$ & $S X=95$ \\
\hline & & & & $p=0.267$ & $p=0.045$ \\
\hline \multirow[t]{3}{*}{ Thurnher et $a^{6}$} & 337 & T1a & $\mathrm{RT}=108$ & - & $\mathrm{RT}=84.3$ \\
\hline & & & Conventional SX = 148 & & Conventional SX = 91.9 \\
\hline & & & Laser excision $=81$ & & $\begin{array}{l}\text { Laser excision }=100 \\
p<0.0001^{*}\end{array}$ \\
\hline Our study & 66 & $\begin{array}{l}\text { Tis, T1a, } \\
\text { T1b, T2 }\end{array}$ & $\begin{array}{l}\text { Laser excision }=61 \\
\text { RT }=5\end{array}$ & $\begin{array}{l}\text { Local control achieved in } \\
95.08 \text { of cases }\end{array}$ & $\begin{array}{l}\text { Laryngeal preservation } \\
\text { achieved in } 98.3 \text { of the cases }\end{array}$ \\
\hline
\end{tabular}


There has been an ongoing debate over the use of laser vs the use of cold microlaryngeal instruments for endoscopic resection. Laser provides the advantage of precise excision and simultaneous hemostasis. Minimizing the healthy tissue removal has become the cornerstone of "phonomicrosurgical" approach to vocal cord cancer. ${ }^{12}$ However, laser can cause thermal damage to deeper structures leading to fibrosis of underlying superficial lamina propria. This fibrosis diminishes mucosal pliability, which is the key determinate of voice quality if glottal closure is maintained. ${ }^{13}$ The thermal damage caused also obscures the margins which can be a problem for the histopathologist. Thus if the lesion is confined to the epithelium and/or superficial lamina propria, a cold instrument tangential dissection with maximal preservation of underlying substantial lesion progression (SLP) is performed. For lesions which have invaded or replaced most of the SLP, a laser resection is performed.

There is a controversy regarding the best treatment modality for lesions involving the anterior commissure. The region of anterior commissure may be difficult to access endoscopically and these lesions are often understaged as the involvement of thyroid cartilage maybe missed and a true T4 lesion may be treated as T2 resulting in poorer outcomes. Thus, the oncologic safety may be compromised. Rodel et $\mathrm{al}^{14}$ analyzed the impact of anterior commissure involvement on local control of 444 cases of early glottis cancer treated endoscopically. The anterior commissure was involved in 153 cases. They noted a decrease in local control rate in case of anterior commissure involvement for T1a and $\mathrm{T} 1 \mathrm{~b}$ tumors but not for T2a tumors. Steiner et $\mathrm{a}{ }^{15}$ reported that laser surgery is effective despite anterior commissure involvement. According to the consensus statement on management in the UK, patients should be given the choice of transoral laser microsurgical resection (TLMR) for tumors involving the anterior commissure, but advised of the greater chance of adverse voice outcome when the anterior commissure is treated surgically. ${ }^{16}$

In 2007, the European Laryngological Society published a revision of their classification for documentation of the extent of endoscopic laser excision of glottic tumors, to aid with evaluation of oncological and functional outcomes. ${ }^{17,18}$ Depending on the extent of resection, there are six types of cordectomies, subepithelial, subligamental, transmuscular, total, extended total, and anterior commissurectomy.

A systematic review done by Yoo et $\mathrm{al}^{19}$ included one meta-analysis and six primary studies comparing posttreatment voice outcomes in patients with $\mathrm{T} 1$ glottic cancer treated by radiotherapy $v s$ transoral laser surgery. The meta-analysis considered the assessment of vocal outcomes based on acoustic and aerodynamic voice parameters. Six studies performed perceptual voice assessments, one of which used a clinician-rated tool and five of which used patient self-perception tools. Overall, the posttreatment quantitative acoustical and aerodynamic voice measurements favored the radiotherapy group, but the patient perception of voice quality did not demonstrate significant differences between the treatment modalities. Cohen et $\mathrm{l}^{20}$ undertook a meta-analysis of voice handicap index (VHI) results for T1a and T1b carcinomas, including six studies and 299 patients. Eleven mean total scores of 12.9 and 18.5 were observed in surgical and irradiated patients respectively, with a nonsignificant difference. Thus the posttreatment voice quality results seem to be comparable for both the treatment modalities in early glottis cancers. In our study the auditory perceptual analysis was conducted utilizing the Perceptual Voice Profile by Oates and Russell. The voice quality depends upon the type of cordectomy performed. ${ }^{21}$

In our series the laryngeal preservation was achieved in $98.3 \%$ of the cases and local control in $95.08 \%$.

To optimize the vocal outcomes in early glottic cancers, we followed certain key principles. The subepithelial infiltration technique was used to determine the depth of infiltration beyond the superficial lamina propria. Using this technique the superficial lesions were addressed by the microflap technique or laser, paying special attention to preserving the superficial lamina propria. Lesions involving vocal ligament and vocalis muscle were resected by laser. A narrow margin resection was done (1-2 mm margin) and free margins were taken from the patients' side and were confirmed on frozen section. Superpulse Acublade laser allows for precise incision and depth penetration. Minimal heat buildup in tissue reduces thermal damage and ensures better tissue margins.

Many studies have shown equal results of voice and local control with both radiotherapy and surgery. The issue today is vocal outcomes. Keeping that in mind, depth becomes as important a parameter for voice as anterior commissure involvement. In our view, type IV cordectomy results in a total loss of muscle and does not allow for a postoperative serviceable voice and there is also less scope of medialization, and thus we feel that for lesions requiring total cordectomy, radiotherapy is a better option for posttreatment voice outcome.

Thus we recommend:

- Subepithelial infiltration technique to assess the depth of penetration of the lesion in superficial lamina propria

- Depth estimation during surgery which can be done by dissecting from periphery to center or by cutting through the tumor (i.e. center to periphery) 
- Cold steel/laser resection for type I cordectomies and laser resection for type II cordectomies and more

- Radiotherapy should be considered as a treatment modality instead of total cordectomy in patients in whom the depth of penetration of lesion is into more than half of the vocalis muscle layer, so as to get postoperative servicable voice

- Anterior commissure involvement is not a contraindication for endoscopic surgery as long as good exposure can be obtained

- Thyroid cartilage involvement may not be a contraindication for a very experienced surgeon.

Thus keeping these principles in mind optimal oncologic as well as voice outcomes can be achieved in early glottic cancer if depth is not up to half the muscle mass.

\section{CONCLUSION}

In our experience, early glottic cancers can be effectively treated by TLMR with good oncologic outcome. For good vocal outcome, it is critical to know the depth of infiltration of the lesion. In our study, near-normal conversational voice to moderate change in voice was found postoperatively after type I, II and III cordectomies. Surprisingly, type VI cordectomy also gave a serviceable vocal outcome with moderate change in voice. Type IV cordectomy does not give a serviceable vocal outcome even if it is performed unilaterally. Thus, radiotherapy should be strongly considered in such cases for better vocal outcomes.

\section{REFERENCES}

1. Higgins KM, Shah MD, Ogaick MJ, Enepekides D. Treatment of early-stage glottic cancer: meta-analysis comparison of laser excision versus radiotherapy. J Otolaryngol Head Neck Surg 2009 Dec;38(6):603-612.

2. Groome PA, O'Sullivan B, Irish JC, Rothwell DM, Math KS, Bissett RJ, Dixon PR, Eapen LJ, Gulavita SP, Hammond JA, et al. Glottic cancer in Ontario, Canada and the SEER areas of the United States. Do different management philosophies produce different outcome profiles? J Clin Epidemiol 2001 Mar;54(3):301-315.

3. Osborn HA, Hu A, Venkatesan V, Nichols A, Franklin JH, Yoo JH, Ceron M, Whelan F, Fung K. Comparison of endoscopic laser resection versus radiation therapy for the treatment of early glottis carcinoma. J Otolaryngol Head Neck Surg 2011 Jun;40(3):200-204.

4. Mahler V, Boysen M, Brondbo K. Radiotherapy or $\mathrm{CO}_{2}$ laser surgery as treatment of T1a glottic carcinoma? Eur Arch OtoRhino-Laryngology 2010 May;267(5):743-750.

5. Schrijvers ML, van Riel EL, Langendijk JA, Dikkers FG, Schuuring E, van der Wal JE, van der Laan BF. Higher laryngeal preservation rate after $\mathrm{CO}_{2}$ laser surgery compared with radiotherapy in T1a glottic laryngeal carcinoma. Head Neck 2009 Jun;31(6):759-764.
6. Thurnher D, Erovic BM, Frommlet F, Brannath W, Ehrenberger K, Jansen B, Selzer E, Grasl MC. Challenging a dogma-surgery yields superior long-term results for T1a squamous cell carcinoma of the glottic larynx compared to radiotherapy. Eur J Surg Oncol 2008 Jun;34(6):692-698.

7. Lynch RC. Suspension laryngoscopy and its accomplishments. Ann Otol Rhinol Laryngol 1915 Sep;24(3):429-478.

8. Stutsman AC, McGavran MH. Ultraconservative management of superficially invasive epidermoid carcinoma of the true vocal cord. Ann Otol Rhinol Laryngol 1971 Aug;80(4):507-512.

9. Nassif R, Loughran S, Moyes C, MacKenzie K. Negative pathology following endoscopic resection of T1a squamous carcinoma of the glottis. J Laryngol Otol 2005 Aug;119(8): 592-594.

10. Strong MS, Jako GJ. Laser surgery in the larynx; early clinical experience with continuous $\mathrm{CO}_{2}$ laser. Ann Otol Rhinol Laryngol 1972 Dec;81(6):791-798.

11. Steiner W. Results of curative laser microsurgery of laryngeal carcinomas. Am J Otolaryngol 1993 Mar-Apr;14(2):116-121.

12. Zeitels SM. Premalignant epithelium and microinvasive cancer of the vocal fold: the evolution of phonomicrosurgical management. Laryngoscope 1995 Mar;105(Suppl 3):S1-5.

13. Zeitels SM, Hillman RE, Desloge RB, Mauri M, Doyle PB. Phonomicrosurgery in singers and performing artists: $P$ treatment outcomes, management theories, and future directions. Ann Otol Rhinol Laryngol 2002 Dec;111(Suppl 190):S21-40.

14. Rodel RM, Steiner W, Muller RM, Kron M, Matthias C. Endoscopic laser surgery of early glottic cancer: involvement of the anterior commissure. Head Neck 2009 May;31(5): 583-592.

15. Steiner W, Ambrosch P, Rodel RM. Impact of anterior commissure involvement on local control of early glottic carcinoma treated by laser microresection. Laryngoscope 2004 Aug;114(8):1485-1491.

16. Bradley PJ, Mackenzie K, Wight R. Consensus statement on management in the UK: transoral laser assisted microsurgical resection of early glottic cancer. Clin Otolaryngol 2009 Aug;34(4):367-373.

17. Remacle M, Eckel HE, Antonelli A, Brasnu D, Chevalier D, Friedrich G, Olofsson J, Rudert HH, Thumfart W, de Vincentiis $\mathrm{M}$, et al. Endoscopic cordectomy. A proposal for a classification by the Working Committee, European Laryngological Society. Eur Arch Otorhinolaryngol 2000;257(4):227-231.

18. Remacle M, Van Haverbeke C, Eckel H, Bradley $P$, Chevalier D, Djukic V, de Vicentiis M, Friedrich G, Olofsson J, Peretti G, et al. Proposal for revision of the European Laryngological Society classification of endoscopic cordectomies. Eur Arch Otorhinolaryngol 2007 May;264(5):499-504.

19. Yoo J, Lacchetti C, Hammond JA, Gilbert RW. The Head and Neck Cancer Disease Site Group. Role of endolaryngeal surgery (with or without laser) compared with radiotherapy in the management of early (T1) glottic cancer: a clinical practice guideline. Curr Oncol 2013 Apr;20(2):e132-e135.

20. Cohen SM, Garrett CG, Dupont WD, Ossoff RH, Courey MS. Voice-related quality of life in T1 glottic cancer: irradiation versus endoscopic excision. Ann Otol Rhinol Laryngol 2006 Aug;115(8):581-586.

21. Vilaseca I, Huerta P, Blanch JL, Fernández-Planas AM, Jiménez C, Bernal-Sprekelsen M. Voice quality after $\mathrm{CO}_{2}$ laser cordectomy - what can we really expect? Head Neck 2008 Jan;30(1):43-49. 